https://doi.org/10.48009/2_iis_2006_327-332

\title{
A LONGITUDINAL STUDY OF THE IMPACT OF E-MAIL AND SPAM IN THE CORPORATE WORLD
}

\author{
Wallace A. Wood, Bryant University, wwood@bryant.edu \\ Suhong Li, Bryant University, sli@bryant.edu
}

\begin{abstract}
This study examined the impact of e-mail and spam on employees in the corporate world using longitudinal data collected from 1999 to 2005. It was found that the number of business e-mails an employee receives has increased gradually and the amount of spam received by employees decreased. Moreover, about $60 \%$ of the employees spent less than 1 hour per day answering e-mail and $76 \%$ of them do not consider spam a problem. The results also show that the number of companies having an email system minimizing spam has increased from $20 \%$ in 1999 to $93 \%$ in 2005 and the impact of spam on the individual user may not be as serious as has been reported in the press.
\end{abstract}

Keywords: E-Mail, Spam, Longitudinal Study

\section{INTRODUCTION}

E-mail has become a major communication tool today in organizations $[6,7]$ because it incorporates many qualities of both letters and face-to-face communication [7]. E-mail improves teamwork and information sharing. For example, the "cc" function allows information sharing with multiple-coworkers simultaneously. E-mail also contributes to a paperless business environment as many organizations are using e-mail for corresponding and distributing various documents previously sent through the mail system. As it is almost costless to send out and receive e-mail, today's employees increasingly find themselves receiving too many unnecessary e-mails and spending too much time responding to e-mail. Some argue that the amount of e-mail an employee receives today is so overwhelming as to negate the productivity increases that e-mail is supposed to provide [6].

This situation becomes worse when e-mail is used by many individual/companies worldwide to send unsolicited commercial e-mail, known as spam. An individual e-mail message may be sent to a distribution list containing millions of addresses with the sender expecting that only a tiny number of readers will respond to the message. Because of the volume of spam, it decreases business productivity, irritates/distracts those who must trawl around it, and blocks network traffic. Spam may also carry malicious software including code that opens backdoors for hackers. Therefore, spam has been reported to cost the economy millions in lost business, wasted employee time, and expensive IT security and recovery costs [3]. For example, a Ferris Research study found that spam cost U.S. corporations $\$ 8.9$ billion annually, $\$ 2.5$ billion for European businesses, and another $\$ 500$ million for U.S and European service providers. The same study indicates that spam adds up to $\$ 4$ billion in lost productivity for U.S. businesses each year. Another $\$ 3.7$ billion is a result of companies having to buy more powerful servers and more bandwidth [1]. Evett [4] summarized spam and e-mail statistics in 2004 from a number of reputable sources including: Google, Brightmail, Jupiter Research, eMarketer, Gartner, MailShell, Harris Interactive, and Ferris Research. The results are shown in Table 1. It can be seen that a user normally sent 174 e-mails and received 10 e-mails and 6 spams per day. Sixteen percent of e-mail users said they would change their e-mail addresses due to spam. Moreover, $40 \%$ of all e-mail and $15-20 \%$ of corporate e-mail was considered spam.

To counter the massive increase in spam, companies including Microsoft have developed advanced antispam technologies such as Smart Screen, SenderID, and Computational proof to engage in the battle against spam [5]. Moreover, the Controlling the Assault of Non-Solicited Pornography and Marketing Act (CAN-SPAM) went into effect in January 2004. According to this Act, e-mail marketers must comply with the following rules: label their messages as advertisements, include an Internet-based opt-out feature for the recipient that is active for 30 days; and provide the marketing company's physical address in the message. The nation's largest Internet providers, including America Online, EarthLink, Yahoo, and Microsoft Corp., have filed lawsuits against spammers [1]. 
Table 1. Spam and E-mail Statistics in 2004

\begin{tabular}{|ll|}
\hline & Spam Statistics in 2004 \\
\cline { 2 - 2 } E-mail considered Spam & $40 \%$ of all e-mail \\
Daily Spam e-mails sent & 12.4 billion \\
Daily Spam received per person & 6 \\
Annual Spam received per person & 2,200 \\
Spam cost to all non-corp. Internet users & $\$ 255$ million \\
Spam cost to all U.S. Corporations in 2002 & $\$ 8.9$ billion \\
States with Anti-Spam Laws & 26 \\
E-mail address changes due to Spam & $16 \%$ \\
Estimated Spam increase by 2007 & $63 \%$ \\
Annual Spam in 1,000 employee company & 2.1 million \\
Users who reply to Spam e-mail & $28 \%$ \\
Users who purchased from Spam e-mail & $8 \%$ \\
Corporate e-mail that is considered Spam & $15-20 \%$ \\
Wasted corporate time per Spam e-mail & $4-5$ seconds \\
& E-mail Statistics in 2004 \\
Daily e-mails sent & 31 billion \\
Daily e-mails sent per e-mail address & 56 \\
Daily e-mails sent per person & 174 \\
Daily e-mails sent per corporate user & 34 \\
Daily e-mails received per person & 10 \\
E-mail addresses per person & 3.1 average \\
Cost to all Internet users & $\$ 255$ million \\
\hline
\end{tabular}

However, CAN-SPAM has had little effect on reducing the amount of junk e-mail [3, 5]. For example, seventy-two percent of the 1,400 Internet users surveyed by the Pew Internet and American Life Project in 2004 said that they have seen no decline in the amount of spam they receive at work since the law took effect. As a result, 30\% said that they have reduced their use of e-mail [1]. Other studies even reported increasing spam after the implementation of CAN-SPAM. The National Technology Readiness Survey, produced by Rockbridge Associates Inc. and University of Maryland's Center for Excellence in Service, indicated that the number of spam messages a person received per day increased to 18.5 in 2005 and the average time spent each day deleting them is 2.8 minutes [2]. One spam-filtering company cited in a New York Times article stated that $80 \%$ of its customers' e-mail was spam, up from $60 \%$ at the beginning of 2004 [5]. In addition, 90\% of the spam did not comply with the Act's rules.

The reason for the increased spam is twofold. On the one hand, some criticized the Act because it legalizes certain forms of spam, including provisions that make it easier for spammers to continue their spamsending activities [5]. On the other hand, some people do read and respond to spam, which further 
encourages spammers' endeavors, since a small percentage of responses is enough to justify the cost of sending spam. For example, Table 1 shows that $28 \%$ of people responded to spam and $8 \%$ of them actually purchased something advertised by junk emails in 2004.

The above evidence indicates that e-mail and spam may not be just an annoyance but a serious problem. However, according to the study of Tassabehji and Vakola [7], e-mail and spam is not a problem for end users. They surveyed 600 employees of 50 U.K.based organizations and found that the majority of the participants do not feel flooded by e-mail and do not feel that e-mail increased their workload. Moreover, $20 \%$ of them did not receive any spam. Of those that did, only $20 \%$ of all e-mail received was spam. The limited impact of spam on end users may be because organizations have implemented an infrastructure (such as e-mail filter and anti-spam applications) to reduce spam.

The purpose of the study in this paper is to extend the work of Tassabehji and Vakola [7] by investigating whether the impact of e-mail and spam on employees has changed over the years using data collected for the years 1999-2005. Specifically, the following questions were investigated:

1. Did the number of e-mails an employee received per week change during 1999-2005?

2. Did the percentage of spam an employee received per week change during 2001-2005?

3. Did the time an employee spent each day answering e-mails change during 1999-2005?

4. Did answering e-mail become a problem for employees during 1999-2005?
5. Did the percentage of companies having a function to minimize spam change during 1999$2005 ?$

\section{RESEARCH METHODOLGY}

The data for this study was collected from graduate students enrolled in introductory MBA and MSIS courses taught by one of the authors during the academic years 1999-2005. The students were asked to keep a $\log$ for 3-4 weeks for the e-mail they received at their place of employment and to answer several questions related to e-mail and spam in their companies. A total of 352 usable responses were collected.

\section{RESULTS AND DISCUSSION}

\section{E-mails Employees Received per Week during 1999-2005}

The respondents were asked to indicate the number of e-mails they received at work per week. The respondents were then classified into three groups: receiving less then 100 e-mails per week, receiving 100-300 e-mails per week, or receiving more than 300 e-mails per week. Table 2 shows the results. It can be seen that on the average, around half (46\%) of the respondents received less than 100 e-mails per week, or 20 e-mails per work day. Thirty-nine percent of them received between 100-300 e-mails per week, or 20-60 e-mails per work day. Only a small percentage of them $(14 \%)$ received more than 300 e-mails per week, or more than 60 e-mails per work day.

Table 2. The Number of E-mails Employees Received per Week between 1999-2005

\begin{tabular}{|c|c|c|c|c|}
\hline \multirow{2}{*}{ Year } & \multicolumn{3}{|c|}{$\begin{array}{c}\text { Percentage (Number) of Employees } \\
\text { Who Received Per Week }\end{array}$} & \multirow{2}{*}{ Total } \\
\cline { 2 - 5 } & $<\mathbf{1 0 0}$ e-mails & $\mathbf{1 0 0 - 3 0 0}$ e-mails & >300 e-mails & \\
\hline 1999 & $80 \%(20)$ & $20 \%(5)$ & $0 \%(0)$ & 25 \\
\hline 2000 & $67 \%(12)$ & $11 \%(2)$ & $22 \%(4)$ & 31 \\
\hline 2001 & $58 \%(18)$ & $36 \%(11)$ & $6 \%(2)$ & 55 \\
\hline 2002 & $44 \%(24)$ & $40 \%(22)$ & $16 \%(9)$ & 112 \\
\hline 2003 & $38 \%(43)$ & $47 \%(53)$ & $14 \%(16)$ & 51 \\
\hline 2004 & $39 \%(20)$ & $37 \%(19)$ & $24 \%(12)$ & 60 \\
\hline 2005 & $42 \%(25)$ & $45 \%(27)$ & $13 \%(8)$ & 352 \\
\hline Total & $46 \%(162)$ & $39 \%(139)$ & $14 \%(51)$ & \\
\hline
\end{tabular}


Moreover, Table 1 shows that the percentage of employees receiving less than 100 e-mails per week has dropped during 1999-2005 and the percentage of employees receiving between 100-300 e-mails per week has increased. The percentage of employees receiving more than 300 e-mails fluctuated over the years, starting from 0 in 1999 , increased to $22 \%$ in 2000 , then dropped to $16 \%$ in 2001 , increased to $16 \%$ in 2002 and $24 \%$ in 2004 , then dropped to $13 \%$ in 2005 . It can be concluded that the number of business e-mails an employee receives has increased gradually. However, the percentage of employees receiving a large volume of e-mails at work (more than 300) is low over the years.

\section{Percentage of Spam/Junk E-mail Employees Received per Week during 2001-2005}

The respondents were asked to indicate the number of spam e-mails they received at work per week. The respondents were then classified into three groups: those where less than $10 \%$ of e-mails was spam, those where $10-30 \%$ of e-mails was spam, or those where more than $30 \%$ of e-mails was spam. Years 1999 and 2000 were excluded from the analysis because no data was available. Figure 1 shows the results.

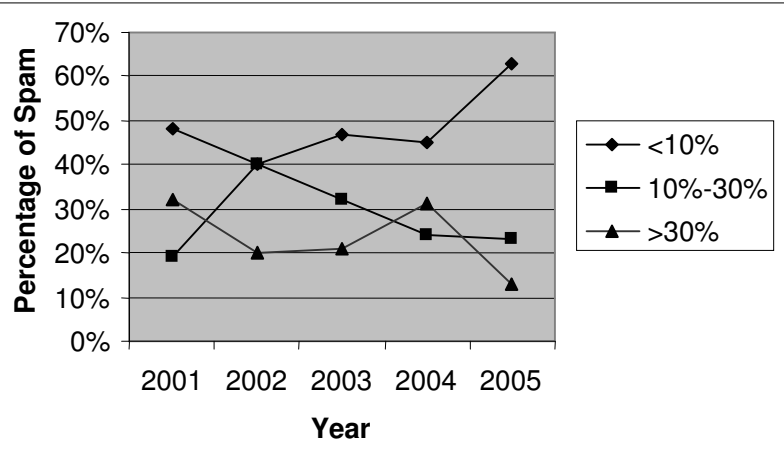

Figure 1. Percentage of Spam Employees Received per Week

Figure 1 shows that roughly half the respondents received less than $10 \%$ spam e-mails during 20012005. Twenty-nine percent of them received between $10-30 \%$ spam and the rest of them $(22 \%)$ received more than $30 \%$ spam. Overall, the percentage of employees receiving less than $10 \%$ spam is consistent at the range of 40-50\% during 2001-2004 and then increased to $63 \%$ in 2005 . The percentage of employees receiving between 10-30\% spam increased to $40 \%$ in 2002 and then gradually decreased through the years 2003-2005. The percentage of employees receiving more than $30 \%$ spam e-mails has decreased gradually from $32 \%$ in 1999 to $13 \%$ in 2005 , except the sudden increase in 2004. Overall, it can be concluded that spam is not a serious problem since around half of the employees received less than $10 \%$ spam consistently during 2001-2005. Moreover, the number of employees receiving less than $10 \%$ spam has increased, while the number of employees receiving more than $10 \%$ spam has decreased over the years, indicating the diminishing impact of spam. This finding is consistent with the finding of Tassabehji and Vakola [7] and in contrast with numerous statistics reported in the literature which consistently show that spam accounts for almost half of e-mail (see Table 1).

There may be several possible explanations for this. First, most of the spam a person receives may come from his/her home e-mail instead of business e-mail since most companies have an e-mail filter or antispam applications to reduce spam, while individuals are less likely to install any application to guard against spam. Moreover, it is known that the cost to a user of free home e-mail usually comes in the form of tons of commercial e-mails. Since e-mail and spam statistics do not always distinguish between home and business e-mail, thus the impact of spam on employees may be over emphasized because of the home factor. Second, anecdotal evidence suggests that spam may be hyped to help security vendors sell more products or services.

\section{The Time Employees Spend Each Day Answering E-mail}

The respondents were asked to indicate the time they spent each day answering e-mail at work. The respondents were then classified into three groups: those spending less than 1 hour answering e-mail, those spending 1-2 hours answering e-mail, and those spending more than 2 hours answering e-mail. Figure 2 shows the results.

It can be seen that the number of the employees spending less than 1 hour per day answering e-mails dropped during 1999-2003 and then increased from 2003 to 2004 , and 2004 to 2005 . The number of the employees spending between 1-2 hours per day answering e-mails increased from 20\% in 1999 to $45 \%$ in 2000 and those spending more than 2 hours dropped from 4\% in 1999 to zero percentage in 2000. Beginning from 2001 to 2005, the number of employees spending between 1-2 hours or more than 2 hours answering e-mails has been consistent across the year with minor fluctuations. Overall, $57 \%$ of the 
participants spent less than 1 hour to answer e-mail each day, $29 \%$ of them spent 1-2 hour answering emails and the rest of them $(14 \%)$ spent more than 2 hours.

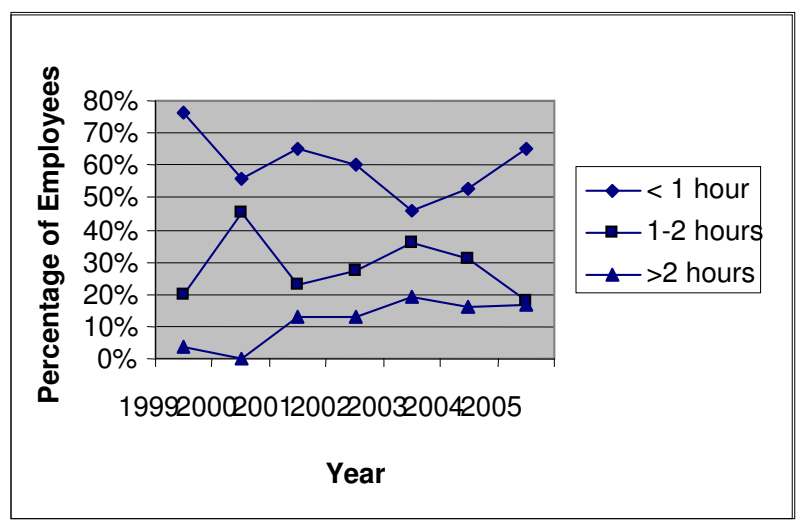

Figure 2. Time Employees Spend Each Day Answering E-mail

\section{Problem in Answering E-mail}

The respondents were asked to indicate whether they consider e-mail a problem at work. The results are shown in Figure 3. Overall, the majority of them do not perceive e-mail creates a problem for them since only $24 \%$ of them said yes that it was a problem during 1999-2005. In 2005, 93\% of the respondents did not consider answering e-mail a problem. This finding is a logical conclusion from our previous analysis, showing that half of employees received less than $10 \%$ spam and about $60 \%$ of employees spent less than 1 hour per day to answer e-mail. Therefore, a majority of them do not consider e-mail a problem.

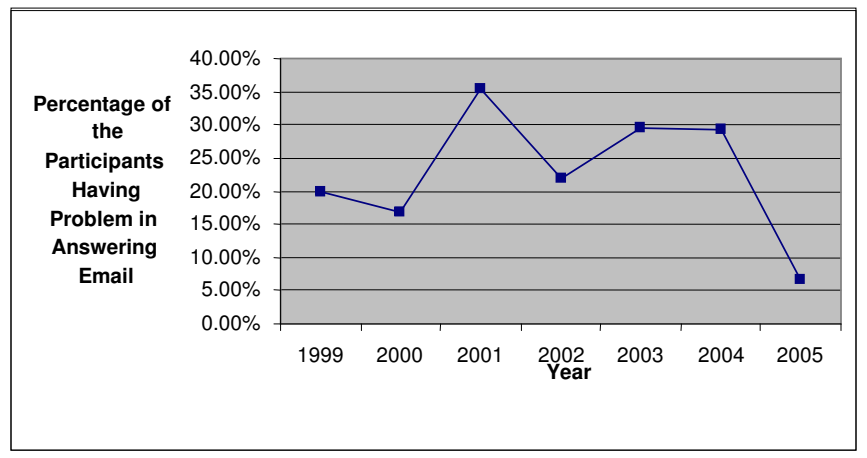

Figure 3. Problem in Answering E-mail

\section{Function to Minimize Spam}

The participants were asked to indicate whether their company e-mail system had a function to minimize spam. Figure 4 show that the number of companies having e-mail systems minimizing spam has increased, from $20 \%$ in 1999 to $93 \%$ in 2005 . As the number of companies having functions to minimize spam increase, we should expect a decrease in spam received by employees. This result again is verified by our previous analysis in relation to spam.

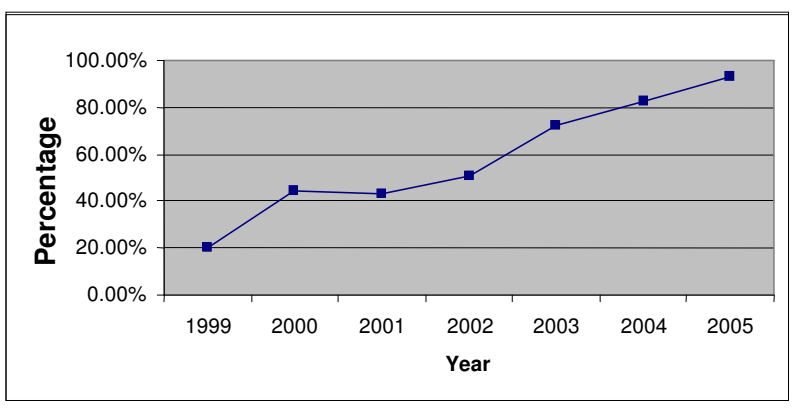

Figure 4. Percentage of Companies Having a Function to Minimize Spam

\section{CONCLUSIONS}

The purpose of this study was to understand the impact of e-mail and spam on employees in the corporate world using the longitudinal data collected from 1999 to 2005 . It was found that the number of business e-mails an employee receives has increased gradually and the amount of spam employees receive decreased from 1999 to 2005. Moreover, about 60\% of the employees spent less than 1 hour per day answering e-mail and $76 \%$ of them did not consider spam a problem. The results also show that the number of companies having an e-mail system minimizing spam has increased over the years, from $20 \%$ in 1999 to $93 \%$ in 2005 . The results indicate that the impact of spam on individual users may not be as serious as what has been reported in the press.

With regard to the period 1999-2005, it should be noticed that the year 2005 stands out. During this year employees were the least troubled by e-mail and spam because the percentages of the surveyed employees receiving more than $30 \%$ spam at work, spending between 1-2 hours answering e-mail each day, and considering e-mail a problem were the lowest, and the percentage of companies (93\%) having a function to minimize spam was the highest. Hopefully, this may indicate that CAN-SPAM has had an effect in fighting spam, which along with 
advanced e-mail filtering and anti-spam applications installed by organizations makes spam less of a problem for individual users of e-mail.

\section{REFERENCES}

1. Anonymous. (May/June 2004). What Spam Law? Next Up...Spim. Information Management Journal, 12-13.

2. Anonymous. (May/June 2005). Deleting Spam Costs Businesses Billions. Information Management Journal, 10.

3. Bland, V. (Aug 2004). Spam is Undermining Business: Six Tips to Beat It. New Zealand Management, 27-32.
4. Evett, D. (2004). Spam Statistics 2004. Retrieved February 14, 2006 from http://spam-filterreview.topten reviews.com/spam-statistics.html.

5. Lee, Y. (2005). The CAN-SPAM Act: A Silver Bullet Solution, Communication of the ACM, 48(6), 131-132.

6. Mendelsohn S., \& Blanton, D. (September/October 2005). Email: Corporate Friend or Foe? AIIM E-DOC Magazine, 36-37.

7. Tassabehji, R, \& Vakola (2005). Business Email: The Killer Impact. Communication of the ACM, 48(11), 64-70. 